\title{
Association of Underlying Comorbidities and progression of COVID-19 Infection Among 2586 Patients Hospitalized in the National Capital Region of India: A Retrospective Cohort Study
}

Rashmi Rana ( $\nabla$ rashmi.rana@sgrh.com )

Sir Ganga Ram Hospital https://orcid.org/0000-0003-0071-089X

Vivek Ranjan

Sir Ganga Ram Hospital

Naveen Kumar

Sir Ganga Ram Hospital

Parul Chugh

Sir Ganga Ram Hospital

Kamini Khillan

Sir Ganga Ram Hospital

Atul Gogia

Sir Ganga Ram Hospital

Devinder Singh Rana

Sir Ganga Ram Hospital

Nirmal Kumar Ganguly

Sir Ganga Ram Hospital

\section{Research Article}

Keywords: Coronavirus disease 2019 COVID-19, diabetes, hypertension, chronic kidney disease, severity, mortality

Posted Date: December 28th, 2021

DOI: https://doi.org/10.21203/rs.3.rs-1184849/v1

License: (9) (1) This work is licensed under a Creative Commons Attribution 4.0 International License. Read Full License 


\section{Abstract}

Objectives This study is conducted to observe the association of diabetes (DM), hypertension (HTN), and chronic kidney disease on the prognosis and mortality of COVID-19 infection in hospital admitted patients.

Methods This is a single centre, observational, retrospective study carried out at Sir Ganga Ram Hospital, Delhi, India. the burden of comorbidities on the prognosis and clinical outcome of COVID-19 patients admitted patients from April 8, 2020, to October 4, 2020. Chi-square and relative risk test were used to observe the association of comorbidities and disease prognosis.

Results A total of 2586 patients were included in the study consisting of $69.6 \%$ of male patients. All the comorbidities were significantly associated with ICU admission and mortality. The relative risk showed that CKD is most prone to severity as well as mortality of the COVID-19 infection followed by HTN and DM. Further with the increase in comorbidity, the risk of ICU admission and mortality increases.

Conclusion Diabetes, hypertension and CKD, all are associated with progression of COVID-19 disease to severity and higher mortality risk. The number of underlying comorbid condition is directly proportional to the progression of disease severity and mortality.

\section{Introduction}

Coronavirus disease 2019 (COVID-19) emerged from Wuhan, China in December 2019 is one of the biggest threats that the entire humankind is facing today [1, 2]. The COVID-19 infection is rapidly spreading and there is a limited knowledge on what would impact the virus critically. Previous studies have reported association of blood groups with susceptibility and mortality of COVID-19 infection [3-5]. Old age and comorbidities have been reported to have poor prognosis of COVID-19 infection. Various studies reported the impact of underlying comorbid conditions of the patients such as diabetes, hypertension, chronic kidney disease (CKD), chronic liver disease (CLD), chronic obstructive pulmonary disease (COPD), cancer, dementia and lung diseases as the major risk factors for COVID-19 disease severity and mortality [6-12].

The risk factors associated with COVID-19 mortality have still not been adequately known. Diabetes is posed to increased risk of hospitalization and intensive care unit (ICU) admission [13, 14]. Diabetes is also common among the patients with fatal outcomes of COVID-19 disease [15-18]. Patients with underlying hypertension are also reported to be associated with poor disease prognosis, longer duration of recovery and mortality $[7,19,20]$. Chronic kidney disease (CKD) another one of the most common comorbidity around the globe is reported to be significantly associated with mortality in COVID-19 patients [21,22]. The clinical outcomes and length of stay are directly related with the underlying comorbid condition of patients [23] Liu et al. found that elderly COVID-19 patients are more susceptible to ICU admission along with higher risk of mortality [24]. Yet the clinical data to guide policy makers and healthcare professional is still insufficient. 
Therefore, it is very crucial to assess any association of comorbidity to establish risk stratification of COVID-19 patient for a better management disease and prevent severe outcomes to decrease the burden on national healthcare systems. The research question of the study is to observe the association of diabetes, hypertension, and chronic kidney disease on the prognosis of COVID-19 infection. Additionally, we investigated the association with age and sex which allows us to identify specific conditions that affects the prognosis of COVID-19 infection.

\section{Methods}

\section{Study Design}

This is a single centre, retrospective study which was carried out at Sir Ganga Ram Hospital, Delhi, India. The study has been reviewed and approved by Ethics Committee of Sir Ganga Ram Hospital.

We investigated the burden of comorbidities on the prognosis and clinical outcome of COVID-19 patients admitted to Sir Ganga Ram Hospital, New Delhi. The impact of COVID-19 and comorbidity was analysed based on the susceptibility to severity of disease (ICU admission), recovery or length of stay at hospital and lastly the mortality. Association was analysed with diabetes mellitus (DM), hypertension (HTN), and chronic kidney disease (CKD). Patients were selected strictly either having underlying condition of DM, HTN and CKD, either alone or in groups of these three conditions. The patients included in this study are RT-PCR confirmed COVID-19 patients admitted from April 8, 2020, to October 4, 2020. The patients were followed up till the time of discharge or decease. Patients with other comorbidities than the abovementioned three were not included in the study.

\section{Data Analysis}

Statistical Package for Social Sciences (SPSS) Version 18 and MedCalc (statistical software) was used for the analysis. Chi square test was used to analyse the distribution of different comorbidities. Relative Risk (RR) test was applied to study the risk of underlying comorbidities of the COVID-19 infected patients. Relative risk is reported with $95 \%$ confidence intervals (Cls). Mann-Whitney test is used to explore the relationship between recovery period (length of stay at hospital) and comorbidities. All the tests are applied as comorbid COVID-19 patients patients vs no comorbid COVID-19 patients. The analysis of multiple comorbid conditions was analysed on comparing any one underlying comorbid condition out of the three mentioned with no comorbid patients. Similarly, any two comorbidities and all the three comorbidities were compared with patients having no comorbidities. Here the association of sex and age groups are not considered, and the association was tested based on the underlying comorbid condition and COVID-19 disease prognosis.

For the analysis of impact of different sex and age group of the COVID-19 patients, the underlying comorbidities are segregated as DM, HTN, CKD, DM+HTN, DM+CKD, HTN+CKD and DM+HTN+CKD, which are compared with non-comorbid COVID-19 patients to understand the precise association of the 
combination underlying conditions and COVID-19 disease prognosis. These combinations of underlying comorbid conditions are analysed while testing the association based on sex and age.

Continuous variables are present as median (IQR) or mean \pm standard deviation. The categorical variables are presented as frequencies (percentages). For analysis 2-tailed test with $p$ value $<0.05$ is considered as statistically significant. Here, we have used chi-square test, Mann-Whitney $U$ test and Relative Risk to analyse the association of underlying comorbidities with severity, recovery duration and mortality of the COVID-19 infected patients.

\section{Results}

Records of COVID-19 infected patients admitted in the hospital were extracted out based on the inclusion and exclusion criteria. A total of 2586 patients were included in the study. Out of $2586,69.6 \%$ of the patients were male. $35 \%$ of the patients were of age greater than equal to 60 years (Table 1). $48.7 \%$ of the COVID-19 infected patients had no underlying comorbidity. Whereas $15 \%$ of the patients had DM, followed by HTN in $10.4 \%$ and CKD in $0.8 \%$ of the patients. The underlying comorbid condition of the patients were further classified on the basis of combination of underlying conditions i.e., DM+HTN, $\mathrm{DM}+\mathrm{CKD}, \mathrm{HTN}+\mathrm{CKD}$ and DM+HTN+CKD in $19.3 \%, 0.6 \%, 1.4 \%$ and $3.8 \%$ respectively (Figure 1 ).

Table 1

Demographic profile of study population $(\mathrm{n}=2586)$

\begin{tabular}{|ll|}
\hline Characteristics & Frequency (\%) \\
\hline Age & $1682(65.0 \%)$ \\
$\geq 60-59(<60)$ & $904(35 \%)$ \\
Sex & $1800(69.6 \%)$ \\
Males & $786(30.4 \%)$ \\
Females & \\
ICU admission & $779(30.1 \%)$ \\
Yes & $1807(69.9 \%)$ \\
No & \\
Outcomes & $2269(87.7 \%)$ \\
Recovered & $317(12.3 \%)$ \\
Deceased & \\
\hline
\end{tabular}




\section{Association of comorbidity with the severity of COVID-19 infection}

The severity of COVID-19 is measured on the basis of admission to ICU. The association of diabetes, hypertension and CKD with susceptibility to severity of COVID-19 infection was analysed with distribution of comorbidity among the COVID-19 infected patients who required ICU admission (COVID+ ICU+) and those who do not required ICU admission (COVID+ ICU-). The patients having diabetic condition was compared to non-diabetic patients irrespective of presence of any other conditions. Similarly, hypertensive and CKD patients were compared to non-hypertensive and non-CKD patients respectively. All the three comorbidities i.e., DM, HTN and CKD showed significant impact with $p$ value $<0.001$ of the underlying comorbid condition in prognosis of COVID-19 disease severity (Table 2). The relative risk of these comorbidities showed that CKD is most prone to severity of the COVID-19 infection followed by HTN and DM (Figure 2). 
Table 2

Distribution of comorbidities between COVID+ICU+ and COVID+ICU-

\begin{tabular}{|c|c|c|c|c|c|}
\hline Comorbidity & $\begin{array}{l}\text { Total } \\
(n=2586)\end{array}$ & $\begin{array}{l}\text { COVID+ ICU+ } \\
(n=779)\end{array}$ & $\begin{array}{l}\text { COVID+ ICU- } \\
(n=1807)\end{array}$ & $p$ value & $\mathrm{RR}(95 \% \mathrm{Cl})$ \\
\hline Diabetes & $1002(38.7 \%)$ & $348(34.7 \%)$ & $654(65.3 \%)$ & $<0.001$ & $1.28(1.14-1.44)$ \\
\hline Yes & $1584(61.3 \%)$ & 431 (27.2\%) & $1153(72.8 \%)$ & & \\
\hline \multicolumn{6}{|l|}{ No } \\
\hline Hypertension & $903(34.9 \%)$ & $333(36.9 \%)$ & $570(63.1 \%)$ & $<0.001$ & $1.39(1.24-1.56)$ \\
\hline Yes & $1683(65.1 \%)$ & $446(26.5 \%)$ & $1237(73.5 \%)$ & & \\
\hline \multicolumn{6}{|l|}{ No } \\
\hline CKD & $170(6.6 \%)$ & $81(47.6 \%)$ & $89(52.4 \%)$ & $<0.001$ & $1.65(1.39-1.95)$ \\
\hline Yes & $2416(93.4 \%)$ & $698(28.9 \%)$ & $1718(71.1 \%)$ & & \\
\hline \multicolumn{6}{|l|}{ No } \\
\hline \multicolumn{6}{|c|}{ Multiple comorbidities } \\
\hline 1 comorbidity & $677(26.2 \%)$ & $206(30.4 \%)$ & $471(69.6 \%)$ & $<0.001$ & $1.20(1.03-1.39)$ \\
\hline No Comorbidity & $1259(48.7 \%)$ & $320(25.4 \%)$ & $939(74.6 \%)$ & & \\
\hline 2 comorbidities & $552(21.3 \%)$ & $203(36.8 \%)$ & $349(63.2 \%)$ & $<0.001$ & $1.45(1.25-1.67)$ \\
\hline No Comorbidity & $1259(48.7 \%)$ & $320(25.4 \%)$ & 939 (74.6\%) & & \\
\hline 3 comorbidities & $98(3.8 \%)$ & $50(51.0 \%)$ & $48(49.0 \%)$ & $<0.001$ & $2.01(1.62-2.49)$ \\
\hline No Comorbidity & $1259(48.7 \%)$ & $320(25.4 \%)$ & $939(74.6 \%)$ & & \\
\hline
\end{tabular}

On comparing the impact of multiple comorbidities with the severity of COVID-19 infection it is found that presence of comorbidity poses greater risk of ICU admission when compared to the patient with no underlying comorbidity. As the number of comorbidities in patients are increased, the risk of severity of COVID-19 infection also increases significantly as observed through the relative risk analyses (Table 2).

\section{Association of comorbidity with the recovery duration of COVID-19 infection}

The median (IQR) recovery duration or the length of stay at the hospital was found to be significantly greater among the patients with comorbidity on applying Mann-Whitney test. Diabetes as well as 
hypertension found to be significantly associated with greater recovery duration. Whereas no association is found with chronic kidney disease. Multiple number of comorbidities in patient are also found to be associated with the length of recovery (Table 3 ).

Table 3

Comparison of comorbid groups distribution and recovery duration (LOS) of patients with COVID-19

\begin{tabular}{|c|c|c|c|c|}
\hline Comorbidity & $\begin{array}{l}\text { Total } \\
(n=2586)\end{array}$ & Median LOS & IQR & $p$ value \\
\hline Diabetes & 1002 (38.7\%) & 10 & $7-15$ & $<0.001$ \\
\hline Yes & $1584(61.3 \%)$ & 9 & $6-12$ & \\
\hline \multicolumn{5}{|l|}{ No } \\
\hline Hypertension & $903(34.9 \%)$ & 10 & $7-15$ & $<0.001$ \\
\hline Yes & $1683(65.1 \%)$ & 9 & $6-12$ & \\
\hline \multicolumn{5}{|l|}{ No } \\
\hline CKD & $170(93.4 \%)$ & 9.5 & $6-17$ & 0.660 \\
\hline Yes & $2416(6.6 \%)$ & 9 & $7-13$ & \\
\hline \multicolumn{5}{|l|}{ No } \\
\hline \multicolumn{5}{|c|}{ Multiple comorbidities } \\
\hline 1 comorbidity & $677(26.2 \%)$ & 10 & $7-14$ & $<0.001$ \\
\hline No Comorbidity & 1259 (48.7\%) & 9 & $6-12$ & \\
\hline 2 comorbidities & $552(21.3 \%)$ & 10 & $7-15$ & $<0.001$ \\
\hline No Comorbidity & $1259(48.7 \%)$ & 9 & $6-12$ & \\
\hline 3 comorbidities & $98(3.8 \%)$ & 10.5 & $6-19$ & $<0.001$ \\
\hline No Comorbidity & $1259(48.7 \%)$ & 9 & $6-12$ & \\
\hline
\end{tabular}

\section{Association of comorbidity with mortality of COVID-19 infection}

To test the association of comorbidity with COVID-19 mortality, comorbidity distribution was compared among deceased and recovered COVID-19 infected patients. Significant difference was found for underlying comorbid condition of the patients. DM, HTN and CKD all are significantly associated with 
mortality among COVID-19 patients. Comparison of multiple underlying comorbid conditions in COVID-19 patients, showed that number of comorbidities are also significantly associated with outcome of the patients. With the increase of underlying comorbid condition of the COVID-19 infected patients, the risk of mortality also increases. CKD is found to be most susceptible to death outcome in the COVID-19 infected patients followed by HTN and lastly DM as shown in Table 4.

Table 4

Distribution of comorbidities among deceased and recovered COVID-19 infected patients

\begin{tabular}{|c|c|c|c|c|c|}
\hline Comorbidity & Total & Deceased & Recovered & $p$ value & $\mathrm{RR}(95 \% \mathrm{Cl})$ \\
\hline Diabetes & 1002 (38.7\%) & $178(17.8 \%)$ & 824 (82.2\%) & $<0.001$ & $2.02(1.65-2.49)$ \\
\hline Yes & $1584(61.3 \%)$ & $139(8.8 \%)$ & 1445 (91.2\%) & & \\
\hline \multicolumn{6}{|l|}{ No } \\
\hline Hypertension & $903(34.9 \%)$ & $176(19.5 \%)$ & $727(80.5 \%)$ & $<0.001$ & $2.33(1.89-2.86)$ \\
\hline Yes & $1683(65.1 \%)$ & $141(8.4 \%)$ & $1542(91.6 \%)$ & & \\
\hline \multicolumn{6}{|l|}{ No } \\
\hline CKD & $170(93.4 \%)$ & $58(34.1 \%)$ & $112(65.9 \%)$ & $<0.001$ & $3.18(2.51-4.04)$ \\
\hline Yes & $2416(6.6 \%)$ & $259(10.7 \%)$ & $2157(89.3 \%)$ & & \\
\hline \multicolumn{6}{|l|}{ No } \\
\hline \multicolumn{6}{|c|}{ Multiple comorbidities } \\
\hline 1 comorbidity & $677(26.2 \%)$ & $73(10.6 \%)$ & $603(89.4 \%)$ & $<0.001$ & $1.46(1.09-1.96)$ \\
\hline No Comorbidity & $1259(48.7 \%)$ & $93(7.4 \%)$ & $1166(92.6 \%)$ & & \\
\hline 2 comorbidities & $552(21.3 \%)$ & $116(21.0 \%)$ & $436(79.0 \%)$ & $<0.001$ & $2.85(2.21-3.67)$ \\
\hline No Comorbidity & $1259(48.7 \%)$ & $93(7.4 \%)$ & $1166(92.6 \%)$ & & \\
\hline 3 comorbidities & $98(3.8 \%)$ & $36(36.7 \%)$ & $62(63.3 \%)$ & $<0.001$ & $4.97(3.59-6.88)$ \\
\hline No Comorbidity & $1259(48.7 \%)$ & $93(7.4 \%)$ & $1166(92.6 \%)$ & & \\
\hline
\end{tabular}

\section{Stratified analysis by age groups}

The COVID-19 infected patients were divided into groups of age $<60$ years and $\geq 60$ years. Chi-square test is applied to compare the association of underlying comorbid conditions with the susceptibility of severity (ICU admission). The association of underlying comorbidities and severity are analysed based on the segregated groups of comorbidities with patients having no comorbidities. The groups are divided as 
DM, HTN, CKD, DM+HTN, DM+CKD, HTN+CKD and DM+HTN+CKD. As the name suggests the group DM, HTN and CKD comprise of patients with only single comorbidity and rest all the comorbid groups have patients having combination of comorbid conditions.

COVID-19 infected patients having diabetes, hypertension or CKD have no association with the severity of disease irrespective of the age of the patients. On analysing the multiple or combination of these comorbidities with severity it was found that among the COVID-19 infected patients of age less than 60 years, $\mathrm{DM}+\mathrm{HTN}$ and $\mathrm{DM}+\mathrm{HTN}+\mathrm{CKD}$ are significantly associated with risk to ICU admission. Whereas underlying $\mathrm{DM}+\mathrm{CKD}$ among $<60$ years patients is almost significantly associated $(p=0.051)$ with higher risk of ICU admission or severity of disease. Among elderly patients of age greater than equal to sixty years, it was found that underlying comorbid conditions does not have any impact in severity of disease except for the patients with all the three comorbidities i.e., DM+HTN+CKD (refer to Figure 2 and Supplementary Table 1).

The analysis of relative risk of the of severity of COVID-19 shows that younger patients with underlying comorbidities are relatively at higher risk of severity of COVID-19 infection compared to the elderly patients with similar underlying condition except for DM (<60 years= RR: $0.98,95 \%$ Cl: 0.76-1.26; $\geq 60$ years= RR: $1.09,95 \% \mathrm{Cl}: 0.82-1.43)$ where the relative risk of ICU admission among patients with age less than $<60$ years is comparatively lower but almost similar to the patients with age $\geq 60$ years (Figure 2).

On comparing the association of mortality with underlying comorbid conditions in COVID-19 patients, it is found that CKD is significantly associated with mortality of the patients regardless of the age of the patients. Among the patients of age $<60$ years, combination of $D M+H T N, H T N+C K D$ and $D M+H T N+C K D$ are at significantly higher risk to mortality on comparing with the non-comorbid COVID-19 patients. Whereas, among the patients of age $\geq 60$ years it is found that patients having all the three comorbidities are associated with mortality due to COVID-19 infection (Supplementary table 2). The relative risk analysis found that patients belonging to younger age group ( $<60$ years) are relatively at higher risk of mortality as compared to older patients ( $\geq 60$ years) with same underlying conditions.

\section{Stratified analysis by sex}

COVID-19 infected males and females were analysed to observe the association of underlying comorbidities and susceptibility of COVID-19 infection to severity of disease and mortality due to infection. COVID-19 infected male patients having HTN, DM+HTN, DM+CKD and DM+HTN+CKD groups are found to be significantly associated to higher risk of disease severity or ICU admission. Whereas, among females DM+HTN and DM+HTN+CKD underlying comorbidities poses a higher risk of severity of disease (Figure 2 and Supplementary Table 3). On comparing the relative risk of ICU admission in COVID19 patients it was observed that females with DM, HTN, DM+HTN, HTN+CKD are more prone to severity of disease and ICU admission as compared to the males with same underlying conditions (Figure 2).

For association of comorbidity with mortality in COVID-19 patients it was found that among females, each comorbid groups are significantly associated with mortality in COVID-19 patients except for 
hypertensive condition. In males no association of comorbidities with mortality was found in DM, HTN, and HTN+CKD patients (Figure 2 and Supplementary Table 4). The relative risk analysis showed that females are at greater risk of mortality as compared to the males having same comorbid conditions except for the hypertensive patients where the relative risk of mortality in females is less than the relative risk of mortality in males (Figure 2).

\section{Discussion}

The current study was undertaken to evaluate the association of comorbidities with prognosis of COVID19 infection. For this study we have considered three most common comorbidities throughout the world i.e., diabetes, hypertension, and chronic kidney disease. Further we have analysed the impact of multiple underlying comorbid conditions on the prognosis of COVID-19 infection. The prognosis of COVID-19 infection is varying, and it suggests that the patients who develop severe illness and severe inflammatory response might experience longer exposure to virus and thereby longer recovery period [25].

Our analysis of COVID-19 infected patients in Delhi, India shows that the underlying comorbid conditions are significantly associated with ICU admission, duration of recovery and mortality. Chronic kidney disease is associated with the highest risk of mortality in COVID-19 infected patients out of the three studied comorbidities, followed by hypertension and the least for diabetes mellitus. On analysing the association of multiple comorbidities, it is found that with the increase in number of comorbidities, the risk of severity of disease as well as the risk of mortality also increases indicating a directly proportional relation with the number of comorbidities.

All the patients of the study population received treatment based on their presenting signs and symptoms as per the attending physician's discretion along with the hospital/institutions protocol based on the national guidelines for the management of COVID-19 of government of India which were changing from time to time. Several different medicines are used for the treatment of these patients which includes anticoagulants, antibiotics, hydroxychloroquine (HCQ), azithromycin, doxycycline, ivermectin, remdesivir, steroids (dexamethasone or methylprednisolone),tocilizumab and oxygen support or ventilators as per the requirement and need of the patient.

The rate of ICU admission among the COVID-19 infected patients is reported to be lower in other countries such as Republic of Korea [26], Mexico [27], America [28, 29], United Arab Emirates [30] and Iran [31] than the present study. Similarly, the mortality rate reported in our study was lower than that reported in other studies from different countries like the United States of America [29, 32], the United Arab Emirates [33]. Whereas other countries like Mexico [27] and Republic of Korea [7, 26] seem to have lower mortality rate than the present study on Indian population.

Cegan et al. performed a cross national study to assess the role of comorbidities and age in progression of COVID-19 infection. It was found that with the increase in number of comorbidities the rate of ICU admission as well as mortality increases [28]. The result from this study is similar to the result of our study which also reported increased percentage of patients admitted to ICU and mortality with increased 
number of underlying comorbid conditions in COVD-19 patients. Our study moves a step further and also calculates the relative risk of ICU admission and mortality with the number of comorbid conditions which also found a higher relative risk with increased number of comorbidities. Another study from UAE observed similar results as of this study and found that comorbidities are associated with higher risk of mortality and observed diabetes, hypertension as well as CKD, all are significantly at higher risk of mortality [34] Diabetes is one of the most reported comorbidities associated with disease progression and mortality of COVID-19 [35-37]. Cho et al., reports DM, HTN and CKD to be significantly associated with mortality of the patients [7]. This study resembles with the results of these mentioned study studies with comorbidities being significantly impacting the disease outcome and mortality due to COVID-19.

In the present study we found that diabetes and hypertension are related to longer recovery period and CKD have no association with the recovery duration in COVID-19 infected patients. In a study from USA by Aldhaeefi et al., it was found that hypertension as well as CKD are associated with longer duration of COVID-19 negativity in patients, whereas no association of diabetes with recovery time in COVID-19 patients was reported [20]. On observing multiple underlying comorbidities in COVID-19 infected patients with patients having no comorbidity, the recovery duration was found to be significant in all the three categories. Further a clarity is missing on the persistent positivity of PCR test for SARS-CoV-2 virus may be due to a live virus or it might be reflecting residual viral RNA from the dead virus.

Sex of the COVID-19 infected patients have been previous reported to affect the disease progression and outcome of COVID-19 infection. Males are reported to be at higher risk of infection than females [38]. This can be further observed in many studies where the number of COVID-19 infected males are as much as twice the number of COVID-19 infected female $[18,33,39,40]$. In our study we found that even though the number of COVID-19 admitted male patients are more than the twice of females. But on calculating the risk ratio of males and females with same underlying comorbid conditions it was observed that the risk of severity of infection and mortality is higher among the females.

Delayed clearance of SARS-CoV-2 or longer duration of recovery is associated with higher mortality rate [34]. Delayed clearance is also found among the elderly patients [40, 41]. In another study by Wang et al. it was found that among the non-survivor ICU patients, nearly $76 \%$ of the patients were of age greater than 60 years. Our study contradicts these results and finds that patients of age less than sixty years are at higher risk of ICU admission as well as mortality compared to the patients of age group $\geq 60$ years with same underlying comorbid conditions [23]. We analysed the association of each comorbidity segregated into each possible different groups that can be categorised among the three comorbidities. The strength of association of these comorbidities decreased with age. The relative risk for mortality was greater for patients with age $<60$ years in contrast to patients with age $\geq 60$ years for all the three comorbidities. The results are similar to that of a retrospective study from Mexico involving COVID-19 patients who had diabetes also showed that the older patients have lower hazard ratio then the patients of young age group [27].

\section{Limitations}


The major limitation of the study is the non-availability of patient's presenting symptoms and the treatment offered to the patient. Further the duration of the study was wide, the treatment guidelines were dynamic which was changing continuously based on the national guidelines for management of COVID19 which might have affected the progression of disease.

\section{Conclusion}

Diabetes mellitus, hypertension and chronic kidney disease, all are associated with progression of COVID19 disease to severity and higher mortality risk. The number of underlying comorbid condition is directly proportional to the progression of disease severity and mortality. In Indian population, adults with age less than 60 years are susceptible to severe disease progression than the older individuals with same underlying comorbid conditions. Lastly, females are at higher risk of disease severity than males of same underlying comorbid conditions.

\section{Declarations}

\section{No data availability statement}

All Datasets generated for this study are included in the article/supplementary materials.

\section{Conflict of interest}

The authors have declared no conflict of interest.

\section{Author contribution}

R.R. and V.R. contributed to the study design and concept. R.R., V.R., A.G. were involved in the study organization. V.R., A.G. and K.K., contributed to the recruitment of patients and collection of data. R.R., N.K. and P.C. contributed to data analysis and data interpretation. R.R. and N.K. wrote the manuscript. Manuscript was edited by D.S.R and N.K.G. The final version of the manuscript was read and approved by all authors.

\section{Acknowledgement}

The authors would like to thank Monika Khanduja for data entry and management. R.R. thankful to Sir Ganga Ram Hospital, Delhi, India for providing the necessary support.

\section{References}

1. Rana R, Tripathi A, Kumar N, Ganguly NK (2021) A Comprehensive Overview on COVID-19: Future Perspectives. Frontiers in Cellular and Infection Microbiology. 11

2. Boni MF, Lemey P, Jiang X, Lam TT, Perry BW, Castoe TA, Rambaut A, Robertson DL (2020) Evolutionary origins of the SARS-CoV-2 sarbecovirus lineage responsible for the COVID-19 pandemic. 
Nature microbiology 5(11):1408-1417

3. Rana R, Ranjan V, Kumar N (2021) Association of ABO and Rh Blood Group in Susceptibility, Severity, and Mortality of Coronavirus Disease 2019: A Hospital-Based Study from Delhi, India. Front Cell Infect Microbiol 11:767771. doi: 10.3389/fcimb

4. Zhao J, Yang Y, Huang H, Li D, Gu D, Lu X, Zhang Z, Liu L, Liu T, Liu Y, He Y (2021) Relationship between the $A B O$ blood group and the coronavirus disease 2019 (COVID-19) susceptibility. Clinical Infectious Diseases 73(2):328-331. doi: 10.1093/cid/ciaa1150

5. Göker H, Karakulak EA, Demiroğlu H, Ceylan ÇM, Büyükaşik Y, Inkaya A, Aksu S, Sayinalp N, Haznedaroğlu IC, Uzun Ö, Akova M (2020) The effects of blood group types on the risk of COVID-19 infection and its clinical outcome. Turkish journal of medical sciences 50(4):679-683. doi: 10.3906/sag-2005-395

6. Sanyaolu A, Okorie C, Marinkovic A, Patidar R, Younis K, Desai P, Hosein Z, Padda I, Mangat J, Altaf M (2020) Comorbidity and its impact on patients with COVID-19. SN comprehensive clinical medicine. $1-8$

7. Cho SI, Yoon S, Lee HJ (2021) Impact of comorbidity burden on mortality in patients with COVID-19 using the Korean health insurance database. Sci Rep 11(1):1-9

8. Guan WJ, Liang WH, Zhao Y, Liang HR, Chen ZS, Li YM, Liu XQ, Chen RC, Tang CL, Wang T, Ou CQ (2020) Comorbidity and its impact on 1590 patients with COVID-19 in China: a nationwide analysis. European Respiratory Journal. 55(5)

9. Solís P, Carreňo H (2020) COVID-19 fatality and comorbidity risk factors among confirmed patients in Mexico. MedRxiv

10. Williamson EJ, Walker AJ, Bhaskaran K, Bacon S, Bates C, Morton CE, Curtis HJ, Mehrkar A, Evans D, Inglesby P, Cockburn J (2020) OpenSAFELY: factors associated with COVID-19 death in 17 million patients. Nature 584(7821):430

11. Nachtigall I, Lenga P, Jóźwiak K, Thürmann P, Meier-Hellmann A, Kuhlen R, Brederlau J, Bauer T, Tebbenjohanns J, Schwegmann K, Hauptmann M (2020) Clinical course and factors associated with outcomes among 1904 patients hospitalized with COVID-19 in Germany: an observational study. Clin Microbiol Infect 26(12):1663-1669

12. Piroth L, Cottenet J, Mariet AS, Bonniaud P, Blot M, Tubert-Bitter P, Quantin C (2021) Comparison of the characteristics, morbidity, and mortality of COVID-19 and seasonal influenza: a nationwide, population-based retrospective cohort study. The Lancet Respiratory Medicine 9(3):251-259

13. Petrilli CM, Jones SA, Yang J, Rajagopalan H, O’Donnell L, Chernyak Y, Tobin KA, Cerfolio RJ, Francois F (2020) Factors associated with hospital admission and critical illness among 5279 people with coronavirus disease 2019 in New York City: prospective cohort study.Bmj.369

14. Ioannou GN, Locke E, Green P, Berry K, O’Hare AM, Shah JA, Crothers K, Eastment MC, Dominitz JA, Fan VS (2020) Risk factors for hospitalization, mechanical ventilation, or death among 10131 US veterans with SARS-CoV-2 infection. JAMA network open 3(9):e2022310 
15. Barron E, Bakhai C, Kar P, Weaver A, Bradley D, Ismail H, Knighton P, Holman N, Khunti K, Sattar N, Wareham NJ (2020) Associations of type 1 and type 2 diabetes with COVID-19-related mortality in England: a whole-population study. The lancet Diabetes \& endocrinology 8(10):813-822

16. Tartof SY, Qian L, Hong V, Wei R, Nadjafi RF, Fischer H, Li Z, Shaw SF, Caparosa SL, Nau CL, Saxena (2020) Obesity and mortality among patients diagnosed with COVID-19: results from an integrated health care organization. Annals of internal medicine 173(10):773-781

17. Yan Y, Yang Y, Wang F, Ren H, Zhang S, Shi X, Yu X, Dong K (2020) Clinical characteristics and outcomes of patients with severe covid-19 with diabetes. BMJ open diabetes research and care 8(1):e001343

18. Stoian AP, Pricop-Jeckstadt M, Pana A, lleanu BV, Schitea R, Geanta M, Catrinoiu D, Suceveanu Al, Serafinceanu C, Pituru S, Poiana C (2020) Death by SARS-CoV 2: a Romanian COVID-19 multi-centre comorbidity study. Sci Rep 10(1):1-1

19. CDC. Coronavirus (COVID-19): symptoms of coronavirus. Centers for Disease Control and Prevention (2020) [Accessed August 18, 2021, https://www.cdc.gov/coronavirus/2019-ncov/symptomstesting/symptoms.html]

20. Aldhaeefi M, Tahir Z, Cote DJ, Izzy S, El Khoury J (2021) Comorbidities and age are associated with persistent COVID-19 PCR Positivity. Frontiers in cellular and infection microbiology. 11:265

21. Parra-Bracamonte GM, Parra-Bracamonte FE, Lopez-Villalobos N, Lara-Rivera AL (2021) Chronic kidney disease is a very significant comorbidity for high risk of death in patients with COVID-19 in Mexico. Nephrology 26(3):248-251

22. Parra-Bracamonte GM, Lopez-Villalobos N, Parra-Bracamonte FE (2020) Clinical characteristics and risk factors for mortality of patients with COVID-19 in a large data set from Mexico. Annals of epidemiology 52:93-98

23. Wang Y, Lu X, Li Y, Chen H, Chen T, Su N, Huang F, Zhou J, Zhang B, Yan F, Wang J (2020) Clinical course and outcomes of 344 intensive care patients with COVID-19. American journal of respiratory and critical care medicine 201(11):1430-1434

24. Liu K, Chen Y, Lin R, Han K (2020) Clinical features of COVID-19 in elderly patients: A comparison with young and middle-aged patients. Journal of Infection 80(6):e14-e18

25. Xie J, Ding C, Li J, Wang Y, Guo H, Lu Z, Wang J, Zheng C, Jin T, Gao Y, He H (2020) Characteristics of patients with coronavirus disease (COVID-19) confirmed using an IgM-IgG antibody test. Journal of medical virology 92(10):2004-2010

26. Song J, Park DW, Cha JH, Seok H, Kim JY, Park J, Chi H (2021) Clinical course and risk factors of fatal adverse outcomes in COVID-19 patients in Korea: a nationwide retrospective cohort study. Sci Rep 11(1):1-1

27. Woolcott OO, Castilla-Bancayán JP (2021) The effect of age on the association between diabetes and mortality in adult patients with COVID-19 in Mexico. Sci Rep 11(1):1-0

28. Cegan JC, Trump BD, Cibulsky SM, Collier ZA, Cummings CL, Greer SL, Jarman H, Klasa K, Kleinman G, Surette MA, Wells E (2021) Can Comorbidity Data Explain Cross-State and Cross-National 
Difference in COVID-19 Death Rates? Risk Management and Healthcare Policy 14:2877

29. Richardson S, Hirsch JS, Narasimhan M, Crawford JM, McGinn T, Davidson KW, Barnaby DP, Becker LB, Chelico JD, Cohen SL, Cookingham J (2020) Presenting characteristics, comorbidities, and outcomes among 5700 patients hospitalized with COVID-19 in the New York City area. Jama 323(20):2052-2059

30. Deeb A, Khawaja K, Sakrani N, AlAkhras A, Al Mesabi A, Trehan R, Kumar PC, Babiker Z, Nagelkerke N, Fru-Nsutebu E (2021) Impact of Ethnicity and Underlying Comorbidity on COVID-19 Inhospital Mortality: An Observational Study in Abu Dhabi, UAE. BioMed Research International. 2021

31. Akhavizadegan H, Aghaziarati M, Roshanfekr Balalemi MG, Arman Broujeni Z, Taghizadeh F, Akbarzadeh Arab I, Janani M (2021) Relationship Between Comorbidity, Chronic Diseases, ICU Hospitalization, and Death Rate in the Elderly with Coronavirus Infection. Iranian Journal of Ageing 16(1):86-101

32. Grasselli G, Zangrillo A, Zanella A, Antonelli M, Cabrini L, Castelli A, Cereda D, Coluccello A, Foti G, Fumagalli R, lotti G (2020) Baseline characteristics and outcomes of 1591 patients infected with SARS-CoV-2 admitted to ICUs of the Lombardy Region, Italy. Jama 323(16):1574-1581

33. Elemam NM, Hannawi H, Al Salmi I, Naeem KB, Alokaily F, Hannawi S (2021) Diabetes mellitus as a comorbidity in COVID-19 infection in the United Arab Emirates. Saudi Medical Journal 42(2):170

34. Zhou F, Yu T, Du R, Fan G, Liu Y, Liu Z, Xiang J, Wang Y, Song B, Gu X, Guan L (2020) Clinical course and risk factors for mortality of adult inpatients with COVID-19 in Wuhan, China: a retrospective cohort study. The lancet 395(10229):1054-1062

35. Zhu L, She ZG, Cheng X, Qin JJ, Zhang XJ, Cai J, Lei F, Wang H, Xie J, Wang W, Li H (2020) Association of blood glucose control and outcomes in patients with COVID-19 and pre-existing type 2 diabetes. Cell Metabol 31(6):1068-1077

36. Guo W, Li M, Dong Y, Zhou H, Zhang Z, Tian C, Qin R, Wang H, Shen Y, Du K, Zhao L (2020) Diabetes is a risk factor for the progression and prognosis of COVID-19. Diabetes/metabolism research and reviews 36(7):e3319

37. Jin JM, Bai P, He W, Wu F, Liu XF, Han DM, Liu S, Yang JK (2020) Gender differences in patients with COVID-19: focus on severity and mortality. Frontiers in public health 8:152

38. Bahardoust M, Heiat M, Khodabandeh M, Karbasi A, Bagheri-Hosseinabadi Z, Ataee MH, Seidalian N, Babazadeh A, Agah S, Abyazi MA (2021) Predictors for the severe coronavirus disease 2019 (COVID$19)$ infection in patients with underlying liver disease: a retrospective analytical study in Iran. Sci Rep $11(1): 1-6$

39. Badedi M, Makrami A, Alnami A (2021) Co-morbidity and blood group type risk in coronavirus disease 2019 patients: A case-control study. J Infect Public Health 14(4):550-554

40. Chen X, Hu Mt W, Yang M, Ling J, Zhang Y, Deng L, Li J, Lundkvist Ã, Lindahl JF, Xiong Y (2021) Risk factors for the delayed viral clearance in COVID-19 patients. The Journal of Clinical Hypertension 23(8):1483-1489 
41. Fu Y, Han P, Zhu R, Bai T, Yi J, Zhao X, Tao M, Quan R, Chen C, Zhang Y, He Q (2020) Risk factors for viral RNA shedding in COVID-19 patients.European Respiratory Journal.56(1)

\section{Figures}

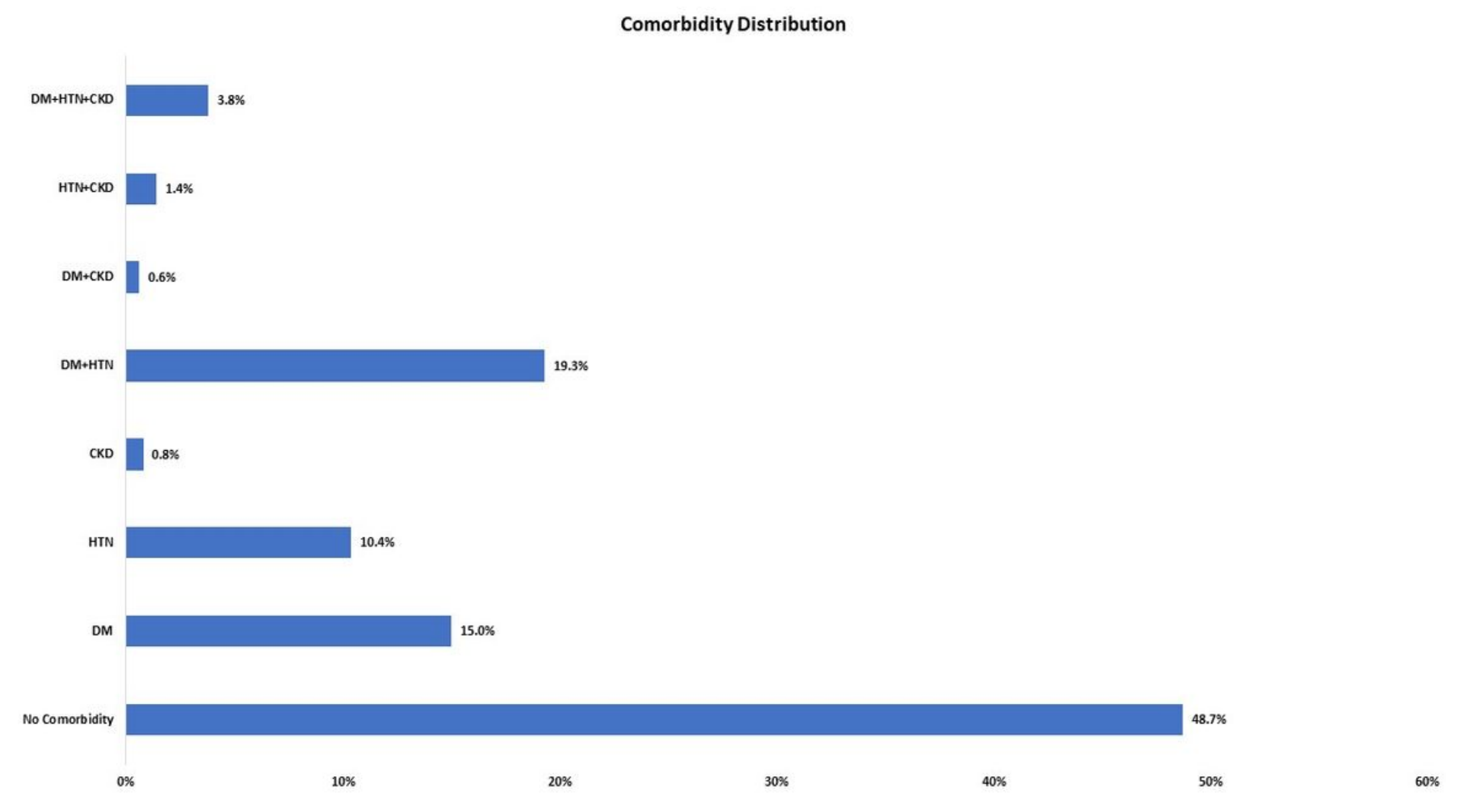

Figure 1

Distribution of underlying comorbid conditions of the study population 


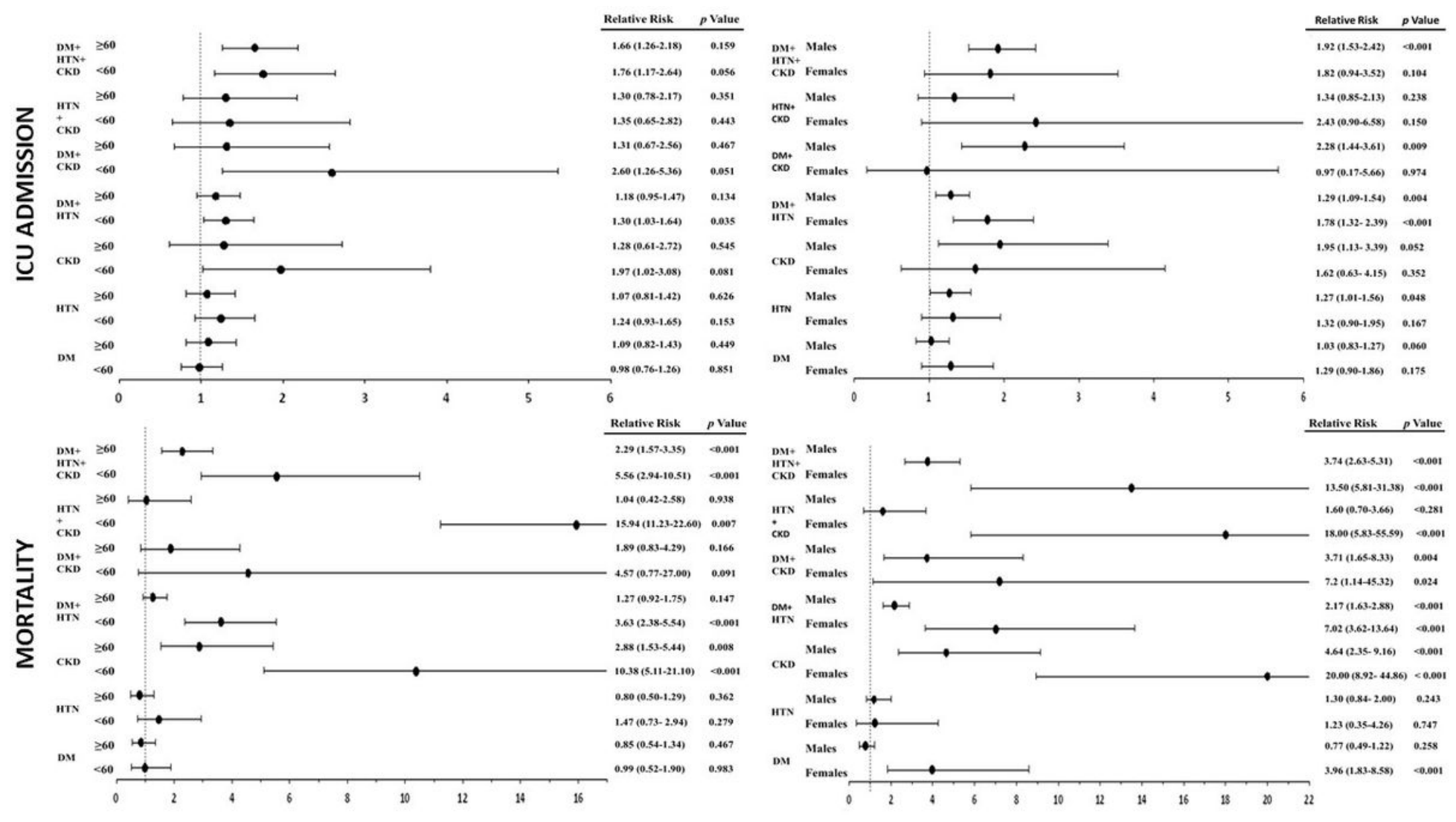

Figure 2

Relative risk distribution of underlying comorbid conditions with prognosis of COVID-19 infection on the basis of age and sex

\section{Supplementary Files}

This is a list of supplementary files associated with this preprint. Click to download.

- SupplementaryTable1.docx

- SupplementaryTable2.docx

- SupplementaryTable3.docx

- SupplementaryTable4.docx 